Relations industrielles

Industrial Relations

\title{
Englewood Cliffs, Occupational information, by C.L. Shartel, Prentice-Hall Inc., Englewood Cliffs, Third Edition, 1959.
}

\section{C.-R. Giroux}

Volume 15, numéro 2, avril 1960

URI : https://id.erudit.org/iderudit/1022041ar

DOI : https://doi.org/10.7202/1022041ar

Aller au sommaire du numéro

Éditeur(s)

Département des relations industrielles de l’Université Laval

ISSN

0034-379X (imprimé)

1703-8138 (numérique)

Découvrir la revue

Citer ce compte rendu

Giroux, C.-R. (1960). Compte rendu de [Englewood Cliffs, Occupational information, by C.L. Shartel, Prentice-Hall Inc., Englewood Cliffs, Third Edition, 1959.] Relations industrielles / Industrial Relations, 15(2), 276-277.

https://doi.org/10.7202/1022041ar

Tous droits réservés (C Département des relations industrielles de l’Université Laval, 1960
Ce document est protégé par la loi sur le droit d'auteur. L’utilisation des services d'Érudit (y compris la reproduction) est assujettie à sa politique d'utilisation que vous pouvez consulter en ligne.

https://apropos.erudit.org/fr/usagers/politique-dutilisation/ 
tionnellement bien disposé ou mal disposé envers votre travail. Quel fait a provoqué cette situation? Qu'elle a été votre attitude par la suite? Quel effet votre attitude a-t-elle eu sur votre travail ? Les entrevues furent analysées selon la méthode d'analyse du contenu de Berelson. Le contenu de l'entrevue est séparé en catégories. Toutefois cette distribution permet de conserver la séquence ou mieux le lien individuel entre les facteurs, les attitudes et les effets.

Les résultats de cette analyse sont des plus révélateurs et des plus lumineux.

Ils viennent confirmer les hypothèses émises au début de l'ouvrage. Un groupe de facteurs se rapportent au continuum de satisfaction: reconnaissance, responsabilité, possibilité de croissance, succès au travail, le contenu du travail. Lorsque ces facteurs produisent un sentiment de satisfaction, ceci a pour effet de maintenir l'individu dans un état d'équilibre ou d'augmenter sa motivation et en même temps d'améliorer sa performance: il est très important de remarquer que ces facteurs relèvent du contenu même de la fonction de l'individu.

Le deuxième groupe de facteurs continuum de mécontentement contribue surtout à la dissatisfaction des employés: politique de compagnie, administration, qualité de la supervision, salaire, relations interpersonnelles, conditions de travail. Il est à remarquer que tous ces facteurs s'apparentent aux conditions de travail, ils sont concomitants à la fonction elle-même. Quand les employés sont satisfaits de ces conditions de travail, ils demeurent dans un état neutre ou état d'équilibre, la motivation n'est pas augmentée. Le mécontentement peut rompre l'équilibre mais en produisant des réactions négatives i.e., réduction de la production, etc. Les auteurs les ont appelé facteurs d'hygiène alors que les premiers facteurs sont reconnus comme facteurs de motivation. Les facteurs de motivation sont propres à l'individı et évoquent chez lui le besoin de trouver dans son occupation une source de croissanice: \& self actualization - ego - involvement ». Une telle révélation replace le syndrome de la motivation industrielle dans une perspective toute nouvelle.

$\mathrm{Par}$ suite des expérienres de Hawthorne et des études sur la dynamique des groupes de l'Université du Michigan (d'ailleurs de si nombreuses investigations n'ont pas tellement produit de résultats tangibles) l'industrie s'est lancée dans des programmes de relations humaines, de développement de la gérance, etc., tout ceci aux fins de produire des attitudes positives et d'augmenter ainsi la production. Le désenchantement fut cruel pour plusieurs, car les employés, quoique n'étant plus mécontents, n'en demeuraient pas moins assez neutres. En effet, tous ces programmes touchaient surtout aux facteurs d'hygiène et ignoraient le contenu du travail comme tel. Il n'est pas étonnant de constater qu'il a été difficile de trouver une relation statistique entre le moral et la performance.

A la suite de Hersey, Argyris, Taylor, Lawshe, etc., les auteurs ont démontré qu'il faut permettre autant que possible à l'individu de contrôler son travail, de faire quelque chose de personnel. D'ailleurs cette conception ne découle-telle pas directement de la nature humaine; que l'on se place du point de vue philosophique ou biologique, l'individualisme est un fait.

L'industrie moderne est une faillite du point de vue humain: d'un côté, la simplification du travail entreprise par la gérance appelle le conformisme en tuant toute initiative; de l'autre, le syndicat en prônant le concept sacré de l'ancienneté ignore trop souvent la compétence humaine.

Ce petit volume de cent cinquante pages est peut être la publication la plus importante à date sur la motivation de l'homme en tant qu'employé ou ouvrier. La survivance de la démocratie moderne ne peut commencer que par une réévaluation de nos valeurs sociales et individuelles. Cette transformation devra s'opérer d'abord dans le milieu du travail, puisque l'homme doit oeuvrer pendant une majeure partie de sa vie.

\section{C.-R. Giroux}

Englewood Cliffs, Occupational information, by C.L. Shartel, Prentice-Hall Inc., Englewood Cliffs, Third Edition, 1959.

L'orientation professionmelle tient maintenant une place importante dans notre système d'éducation. L'une des. 
phases de l'orientation professionnelle consiste à fournir aux personnes intéressées des informations précises sur les groupes d'occupations et sur la nature des occupations. D'où, la nécessité d'organiser un centre de dissémination de l'information, qui reste à la fois assez complet et flexible. Le présent ouvrage a justement comme but d'aider l'orienteur professionnel dans l'aménagement d'une bibliothèque ou centre d'informations sur les occupations.

Cette troisième édition a bien évolué depuis la première et même la deuxième édition. Après un chapitre sur l'utilité et la nécessité des informations occupationnelles, l'auteur fait un exposé démographique du milieu du travail: accroissement et distribution de la population, des employés industriels, structure des occupations, opportunités, etc. Ensuite, lauteur considère les diverses occupations dans leur contexte social. Il est intéressant de noter que la valeur sociale ou le prestige d'une position n'est pas déterminé nécessairement par le contenu de la position ou par sa responsabilité sociale.

Peu de changements ont été apportés au chapitre exposant les diverses méthodes utilisées dans l'analyse et la description des positions. Il aurait été intéressant d'y trouver d'autres méthodes d'analyse des tâches.

Un bref chapitre traite des exigences des positions. Les exigences d'embauchage quoique déterminées par l'employeur sont souvent à la merci des conditions économiques et du marché du travail. Shartle décrit brièvement les examens utilisés pour vérifier si l'applicant rencontre les exigences de la position: examens théoriques, tests psychologiques, etc.

Quoiqu'il n'y ait pas de chapitre spécialement réservé à l'organisation d'une bibliothèque et d'un centre d'informations, il $y$ a suffisamment de suggestions dans le texte pour guider le conseiller d'orientation. Shartle cite de nombreuses sources d'où il est possible d'obtenir de l'information occupationnelle.

Par suite de l'importance croissante des carrières militaires, l'auteur leur consacre tout un chapitre.
Shartle a retenu l'orientation générale du volume, mais il en a amélioré le contenu, en tenant compte des exigences sociales et économiques actuelles.

\section{C.-R. GrRoux}

The supervision of Personnel, by John Pfiffner, Englewood Cliffs, PrenticeHall, 1959.

Quel lecteur l'auteur veut-il atteindre avec ce volume?

Le contenu du volume est beaucoup trop simplifié pour s'adresser à des universtitaires «graduate students 》. D'autre part il est trop compliqué pour les contremaîtres et les surveillants car Pfiffner y traite de validité, fiabilité, corrélations et même d'analyse factorielle. Les administrateurs du personnel et les collégiens «undergraduate students » comprendront assez bien les problèmes qui y sont exposés.

La thèse principale de l'auteur est celle-ci: «Irrespective of class, casts, or occupation, both as individuals and groups, people reacts similarly to particular types of management situations » - En d'autres termes, devant certaines situations les gens réagissent de façon semblable par conformisme. Pfiffner a été fortement influencé par les expériences de Hawthorne et par les recherches de l'Université du Michigan. Tout au long du volume, l'auteur sou tien sa thèse en proposant de tout faire pour maintenir et même si possible augmenter le moral des employés. Sa définition opérationnelle du moral telle que donnée par l'auteur, est fortement discutable car on peut déduire qu'elle prône la manipulation des employés pour atteindre les buts de l'organisation.

«High morale is a complex combination of many factors that make people do what the organization expects them to do. Inversely, low morale is a combination of factors that prevent or deter people from doing what the organization expects them to do ». Pour élever le moral, le contremaître doit appliquer la technique non directive de la méthode clinique. Il doit s'occuper de chaque employé individuellement. Aussi, il faudrait que le contremaître soit entraîné à utiliser la méthode clinique, à diagnostiquer les 